\title{
Gold nanoparticle-mediated delivery of paclitaxel and nucleic acids for cancer therapy (Review)
}

\author{
ZHIGUANG WANG $^{1 *}$, JIANYU DONG $^{2 *}$, QIAOJIAJIE ZHAO ${ }^{3}$, YING YING $^{4}$, LIJIE ZHANG $^{3}$, \\ JUNRONG ZOU ${ }^{5}$, SHUQI ZHAO ${ }^{3}$, JIUJU WANG ${ }^{3}$, YUAN ZHAO ${ }^{3}$ and SHANSHAN JIANG ${ }^{3}$ \\ ${ }^{1}$ Frontier Institute of Science and Technology, State Key Laboratory for Mechanical Behavior of Materials, \\ Xi'an Jiaotong University, Xi'an, Shaanxi 710054; ${ }^{2}$ Breast Center, Department of General Surgery, Nanfang Hospital, \\ Southern Medical University, Guangzhou, Guangdong 510515; ${ }^{3}$ Institute of Hematological Research, \\ Shaanxi Provincial People's Hospital, Xi'an, Shaanxi 710000; ${ }^{4}$ Jiangxi Province Key Laboratory of Tumor Pathogens and \\ Molecular Pathology and Department of Pathophysiology, School of Basic Medical Sciences, \\ Nanchang University Medical College, Nanchang, Jiangxi 330006; ${ }^{5}$ Institute of Urology, \\ The First Affiliated Hospital of Gan'nan Medical University, Ganzhou, Jiangxi 341001, P.R. China
}

Received April 26, 2020; Accepted September 18, 2020

DOI: $10.3892 / \mathrm{mmr} .2020 .11580$

\begin{abstract}
Paclitaxel is a potent antineoplastic agent, but poor solubility and resistance have limited its use. Gold nanoparticles (AuNPs) are widely studied as drug carriers because they can be engineered to prevent drug insolubility, carry nucleic acid payloads for gene therapy, target specific tumor cell lines, modulate drug release and amplify photothermal therapy. Consequently, the conjugation of paclitaxel with AuNPs to improve antiproliferative and pro-apoptotic potency may enable improved clinical outcomes. There are currently a number of different AuNPs under development, including simple drug or nucleic acid carriers and targeted AuNPs that are designed to deliver therapeutic payloads to specific cells. The current study reviewed previous research on AuNPs and the development of AuNP-based paclitaxel delivery.
\end{abstract}

\section{Contents}

1. Introduction

2. Mechanisms of paclitaxel action and drug resistance

3. Development of AuNPs for antineoplastic drug delivery

4. Further innovations

Correspondence to: Dr Shanshan Jiang, Institute of Hematological Research, Shaanxi Provincial People's Hospital, 256 West Youyi Road, Xi'an, Shaanxi 710000, P.R. China

E-mail: ji.ang.shan.shan@163.com

${ }^{*}$ Contributed equally

Key words: paclitaxel, gold nanoparticles, cancer, drug delivery, photothermal therapy
5. Limitations

6. Conclusion

\section{Introduction}

Cancer is the second most common cause of mortality worldwide, and lead to an estimated 18.1 incident cases and 9.6 million deaths in 2018 (1). Currently, surgery and cytotoxic chemotherapy are the primary treatments for cancer. However, $70 \%$ of cancer mortality occurs in low- or middle-income countries, which is due to delays in diagnosis and cancer being at a late stage at first presentation (2). Curative surgical resection may no longer be an option in such cases, leaving chemotherapy as the only treatment option (1-3). The global disease burden attributed to cancer underscores the importance of developing chemotherapeutic regimens with enhanced safety, tolerability and efficacy (3).

Paclitaxel is a first-line chemotherapeutic agent for solid tumors, but its use has been confounded by poor solubility, toxicity and the emergence of resistance during therapy $(4,5)$. In order to improve efficacy and to decrease the emergence of resistance, clinicians combine paclitaxel with cisplatin and other antineoplastic drugs (6,7). As nanotechnology has progressed, multiple types of paclitaxel nanoparticles (NPs) have been developed to modulate drug release, promote drug encapsulation, improve bioavailability and target cancer cells by using frame materials of polyethylene glycol, polylactic acid, polyglycolin acid or liposomes (8). Examples include abraxane, which was synthesized by attaching six or seven paclitaxel molecules to albumin NPs of 130-nm diameter, and is considered to be among the most successful nanotherapeutics, as it has been approved as a first-line treatment for non-small cell lung carcinoma by the US Food and Drug Administration (8). Folic acid (FA)-poly NPs have been prepared to target ovarian tumor tissue, and NPs that carry rituximab and paclitaxel have been designed to target CD20-positive B-cell lymphoma (9). 
Gold NPs (AuNPs) have received increasing attention as drug delivery vehicles for cancer therapeutics as they can be engineered to obviate drug insolubility, carry nucleic acid payloads for gene therapy, target specific tumor cell lines, modulate drug release and amplify photothermal therapy (PTT) $(10,11)$. The development of gene therapy has generated increasing interest in the potential of AuNPs to deliver therapeutic nucleic acid payloads $(10,11)$. AuNPs have been designed to carry p53, vascular cell adhesion molecule-1 and other mRNAs $(10,11)$. Furthermore, poly(thymine)-functionalized AuNPs have been synthesized to target mRNA translation using a pcDNA6 vector expressing a bovine growth hormone polyadenylation signal (12). Consequently, it is important to investigate the potential of AuNPs to enhance paclitaxel drug delivery and gene therapy (12). In the current review, the role of paclitaxel in clinical oncology, and previous research on AuNPs and the development of paclitaxel AuNP-based drug delivery and its limitations are examined.

\section{Mechanisms of paclitaxel action and drug resistance}

Paclitaxel, which is isolated from Pacific Yew tree Taxus brevifolia, has been indicated to be a potent antimitotic agent in solid tumor cell lines (13). In 1992, paclitaxel was approved for ovarian cancer treatment by the US Food and Drug Administration (14). According to the National Comprehensive Cancer Network guidelines, paclitaxel is still a first-line chemotherapeutic, is combined with cisplatin, atezolizumab and other anti-neoplastic agents to treat lung, breast, gastric and colorectal cancer, and is also incorporated into pre- and post-operative chemoradiation protocols (15-18).

The antimitotic activity of paclitaxel is mediated via its disruption of microtubular function (19). Paclitaxel promotes the assembly of microtubules from tubulin dimers and stabilizes microtubules by preventing depolymerization (20). This inhibits the reorganization of the microtubule network, which is essential for interphase and mitotic cellular function (20). The polymerization of tubulin dimers decreases their concentration below that required for spindle assembly, thus arresting the cell cycle at the M/G2 phase (21-23). In addition, paclitaxel promotes tumor apoptosis by upregulating death receptor signaling and mitochondrial apoptotic pathways and increases beclin-1-mediated autophagy $(21,24)$. These mechanisms of action have contributed to the emergence of paclitaxel as a first-line antineoplastic drug.

With repeated use, resistance commonly emerges during cancer therapy, and there are three major resistance mechanisms governing this resistance. The first, metabolic inactivation via the cytochrome P450 (CYP) system, was identified in 1994 (25). CYP3A and CYP2C were revealed to serve major roles in paclitaxel metabolism (26). It has also been demonstrated that paclitaxel treatment induces overexpression of CYP2C8 and CYP3A4/5, which is mediated by a nuclear receptor in multiple types of tumor cells, such as pancreatic ductal adenocarcinoma (PDAC), pancreatic adenocarcinoma 2 (PACO2) and PACO7, which are two primary PDAC cells propagated from immune-deficient NOD.Cg-Prkd ${ }^{\text {scid }} I l 2 r g^{\text {tm } 1 \mathrm{Wj}}$ mice, leading to cell-autonomous detoxification (27). A second mechanism behind resistance is the overexpression of ATP binding cassette (ABC) drug efflux transporters. ABCB1, $\mathrm{ABCC} 1, \mathrm{ABCC} 2$ and $\mathrm{ABCG} 2$ have been implicated in paclitaxel resistance in vivo and in vitro (28). ABCB1 and ABCC3 exhibit prominent activity in breast cancer resistance (28). A third mechanism for resistance is associated with drug transport that is mediated by solute carrier proteins. Changes in expression levels of solute carrier proteins may alter drug uptake and determine chemotherapeutic efficacy (29). Single nucleotide polymorphisms in genes that encode solute carriers (SLCs, including SLC31A2, SLC43A1, SLC35A5, SLC41A2 and $S L C O 1 B 3$ ) are associated with paclitaxel sensitivity and resistance in vitro (29). In addition, tumor suppressor genes such as BRCA1, TP53, PTEN, adenomatous polyposis coli, Cyclin Dependent Kinase Inhibitor 1A/2A, High in normal-1 and Bax also affect paclitaxel resistance $(30,31)$. Furthermore, low solubility also limits the use of paclitaxel and all of the aforementioned factors decrease its efficacy. Therefore, it is essential to identify carriers to increase paclitaxel delivery and overcome insolubility and resistance. AuNPs have a number of advantages, including large surface area for drug and nucleic acid binding, non-toxicity and a unique property of amplifying PTT (10-12).

\section{Development of AuNPs for antineoplastic drug delivery}

In the past decade, NPs have been evaluated as novel drug delivery systems. The majority of these particles have a feature size $<200 \mathrm{~nm}$, which helps to overcome the mucus barrier, resulting in low reactogenicity and immunogenicity (32). A previous study demonstrated that NPs $>200 \mathrm{~nm}$ in size induce a mucosal immune response and are taken up by dendritic cells, which secrete inflammation-associated factors, resulting in an immune response (33). In addition, NP carriers exhibit multiple pharmacokinetic advantages, including high loading efficiencies of lipophilic drugs such as 5-fluorouracil, which have low water solubility and intracytoplasmic bioavailability, protection of drugs from degradation, increased drug uptake by tumor cells, enhanced drug concentrations in tumor microenvironments, prolonged drug release and targeted delivery $(34,35)$. The use of NPs for targeted drug delivery allows lower systemic drug exposures and minimal side effects (36). As cancer is the second leading cause of mortality worldwide, the future development and use of antineoplastic nanodrugs is essential $(37,38)$.

Currently, there are four types of nanodelivery systems used or in development in oncology: Polymeric, magnetic, metallic and lipid NPs (36). AuNPs are non-toxic, can be easily synthesized into different sizes and shapes, have a large surface area to transport drugs, exhibit good biocompatibility and absorb near-infrared light (NIR) that can be converted to heat to enable focused thermal therapy (39). AuNPs are used in diagnostic imaging, targeted delivery of drugs, radioisotopes and reactive oxygen species-generating enzymes, and plasmonic PTT and photodynamic therapy (40). Within the past year, $>100$ studies of AuNPs for drug delivery were identified. These studies are classified into 3 groups within the current review: Simple drug-carrying AuNPs, simple nucleic acid-carrying AuNPs and targeted (tissue directed) AuNPs. 
Simple drug-carrying AuNPs. AuNPs were evaluated initially as antineoplastic drug carriers in 2004 (41). AuNPs with a diameter of $32 \mathrm{~nm}$ carrying tumor necrosis factor (TNF) have been used to target MC-38 colon carcinoma tumors in vivo, as the majority of TNF AuNPs target tumor cells with little accumulation in the liver and other organs in mice (41). Furthermore, TNF-carrying AuNPs have been indicated to be more effective against tumor cells compared with naive TNF (41). Studies have also been performed on AuNPs featuring optimization of either the particle or the drug payload $(42,43)$. DM1, which is a maytansine analogue, was developed as a microtubulin inhibitor with the same mechanism of action as paclitaxel, but its toxicity and narrow therapeutic window limited its clinical development. Conjugation of DM1 with ultra-small (diameter, $2 \mathrm{~nm}$ ) AuNPs prolonged the half-life of DM1, increased its cytotoxicity in Bel7404 and HepG2 cells and also improved its tolerability and efficacy in ectopic xenograft models of hepatocellular carcinoma (42). The conjugation of kaempferol, a flavonoid that damages DNA in malignant cells, to 2-nm gold nanoclusters decreases toxicity to normal cells, enhances toxicity to cancer cells and decreases proliferation, colony formation and migration of A549 lung cancer cells (43).

The amplification of PTT is a unique property of AuNPs that facilitates the development of a novel therapeutic modality, combined chemo-PTT (44). Gold nanoshell-coated wedelolactone liposomes combined with NIR-induced hyperthermia release $97.34 \%$ of wedelolactone in $8 \mathrm{~h}$ and exhibit an excellent antitumor efficacy in vivo (44). In conclusion, simple drug-carrying AuNPs are less toxic to normal cells and more toxic to cancer cells.

Nucleic acid-carrying AuNPs. As AuNPs have positive-charged surfaces whereas nucleic acids exhibit negative charges, AuNPs are easily adapted to deliver nucleic acid payloads (45). Small interfering RNAs (siRNAs), mRNAs and micro (mi)RNAs conjugated with AuNPs are used for diagnosis and gene therapy $(45,46)$. Receptor tyrosine kinase-like orphan receptor 1 (ROR1) siRNA-HIV-1 Tat peptide-capped AuNPs have a hydrophilic arginine-rich section that facilitates DNA binding; increases the stability of ROR1 siRNA; enhances cellular uptake and induces apoptosis and necrosis in MDA-MB-231 cells (46).

Furthermore, surfactant-free AuNPs deliver plasmid DNA for tumor treatment (10). AuNPs capped with L-cystine methylester hydrochloride and loaded with p53 plasmid DNA increase p53 expression levels and induce A549 apoptosis, thus inhibiting proliferation without inducing cytotoxicity to normal lung cells (10). The conversion of amine-modified siRNA duplexes into dithiocarbamate ligands followed by conjugation with AuNPs increases siRNA stability and enhances targeted release of siRNA (47). Additional potential advantages of AuNP-based therapy have been demonstrated by the development of an intracellular self-assembly system of DNA and AuNPs (48). In a previous study (48), mRNA of survivin, which is an apoptosis inhibitor, and AuNPs in a complex with its complementary DNA sequence were transfected into cancer cells separately and spontaneously formed cytoplasmic aggregates. Survivin mRNA and AuNPs entered cells easily due to their small size, whereas the larger aggregates exhibited improved intracellular retention. This resulted in enhanced apoptosis and photothermal function, leading to cancer cell death rates of up to $93.3 \%$, with minimal toxicity to normal cells (48).

17-N-allylamino-17-demethoxygeldanamycin (17-AAG) is a heat shock protein 90 (HSP90) inhibitor that blocks ATP binding and inhibits HER2(+) breast cancer cell proliferation (49). However, the anti-tumor effect of 17-AAG is decreased in the absence of Cullin-5 (Cul5), an E3 ubiquitin ligase that is required for HSP90 inhibitor function (49). Consequently, the delivery of 17-AAG and Cul5 DNA via AuNPs can sensitize 17-AAG-resistant breast cancer cells (49). Similarly, poly(sodium 4-styrenesulfonate) and poly(-diallyldimethylammonium chloride) have been used to coat gold nanoprisms carrying a siRNA targeting human program death-ligand 1 (hPD-L1), resulting in the downregulation of hPD-L1 expression and enabling photoacoustic imaging and PTT of lung cancer cells and cell-derived tumors (50).

Zhang et al (51) developed gold nanoshells that are designed for the PTT-stimulated release of siRNA to downregulate HER-2 expression levels and a genetic sequence to express the immunological adjuvant cytosine-guanine motifs to activate anti-tumor immune responses that are mediated by toll-like receptor 9 signaling. This integration of gene therapy, immunotherapy and PTT has been demonstrated to perform well in the treatment of a murine gastric cancer model (51). Consequently, in the presence or absence PTT, AuNPs may be used to carry tumor suppressor genes, oncogene-siRNA and miRNA to inhibit tumor cell proliferation (51).

Targeted AuNPs. In addition to simple AuNPs that are used for drug and nucleic acid delivery and PTT, multifunctional AuNPs have been designed for targeted delivery mediated by $\mathrm{pH}$, short peptides, aptamers, antibodies and receptors (52-57). Glutathione (GSH)-coated $\mathrm{Au}-\mathrm{Fe}_{3} \mathrm{O}_{4}$ nanoshells for doxorubicin (DOX) delivery feature a GSH layer on the nanoshell surface that is attached with an Au-S native bond, which is stable at physiological $\mathrm{pH}$ but rapidly broken down in acidic tumor microenvironments, resulting in local DOX release and cytotoxicity (52). AuNPs coated with biocompatible marine carbohydrate carrageenan oligosaccharide (CAO-AuNPs) have also been designed for $\mathrm{pH}$-dependent drug release. For example, epirubicin (EPI)-CAO-AuNPs release their payload in acidic environments and more easily enter cells via endocytosis, inducing more potent cytotoxicity and increasing apoptosis compared with free EPI (53). A study by Pedrosa et al (53) used polyethylene glycol-coated AuNPs carrying a novel chemotherapeutic candidate $(\mathrm{ZnD})$ and the monoclonal antibody cetuximab that targets epidermal growth factor receptor (EGFR) receptors that are overexpressed in cancer cells, and demonstrated that the addition of cetuximab facilitated both targeting and a second mechanism of action (antagonism of receptor-dependent EFGR signal transduction, resulting in interruption of the cell cycle, apoptosis and inhibition of metastasis and angiogenesis). This candidate was also demonstrated to be active against DOX-resistant tumors in a murine model (54). Afatinib (Afb) is a tyrosine kinase inhibitor that targets EGFR-positive lung cancer, and has disadvantages, including low bioavailability and high toxicity (54). In addition, the native structure of $\mathrm{Afb}$ prevents its direct conjugation to AuNPs. In order to mitigate these issues, an Afb analog containing 


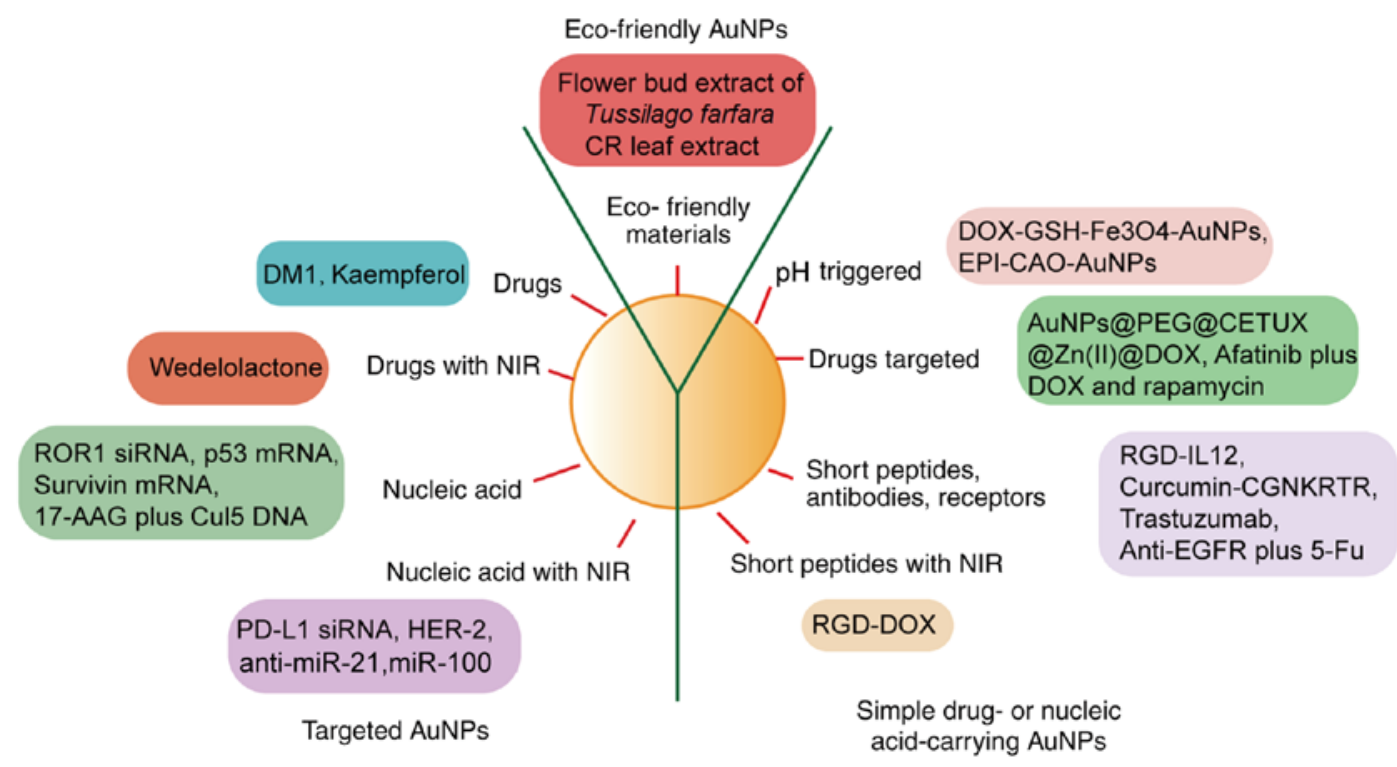

Figure 1. AuNPs are classified into three groups: Simple drug-carrying AuNPs, nucleic acid-carrying AuNPs and multifunctional AuNPs. Eco-friendly AuNPs may be synthesized using environmentally safe materials and production processes. AuNP, gold nanoparticle; NIR, near-infrared; ROR1, receptor tyrosine kinase-like orphan receptor 1; siRNA, small interfering RNA; 17-AAG, 17-N-allylamino-17-demethoxygeldanamycin; Cul5, Cullin5; PD-L1, program death-ligand 1; miR, microRNA; DOX, doxorubicin; GSH, glutathione; EPI, epirubicin; CETUX, cetuximab; RGD, arginylglyclaspartic; IL, interleukin; EGFR, epidermal growth factor receptor; 5-FU, 5-fluorouracil; PEG, polyethylene glycol; CGNKRTR, Nrp-1 receptor-specific short peptide.

a pedant alkynyl has been synthesized (55). The pedant alkynyl is coupled to an azide-functionalized lipoic acid moiety using a copper(I)-catalyzed Huisgen 1,3-dipolar cycloaddition to improve attachment to the AuNP surface with an alkylthiol-gold bond. Afb-AuNPs attenuate pro-inflammatory cytokine release and promote Afb cellular uptake and consequently, Afb-AuNPs exhibit a 3.7-fold increased potency in inhibiting EGFR mutant lung cancer cells, including PC-9 cells (55). Porous silicon NPs in a complex with gold nanorods have been designed for delivery of a three-drug combination of hydrophilic and hydrophobic drugs (Afb, DOX and rapamycin), and the inclusion of Afb, which targets HER-2 and EGFR. This construct confers targeted drug delivery to malignant cells, while gold nanorods facilitate PTT (56). This candidate has demonstrated positive results in vitro and in vivo (56).

The use of short peptides, antibodies and receptors in the design of targeted AuNPs is receiving increasing attention. Paris et al (56) recently investigated a novel strategy of targeting tumor angiogenesis rather than disrupting malignant cells. A tumor vascular endothelium-targeting nanoparticle was designed by incorporating an isoAspGly-Arg peptide that targets $\alpha \beta$-integrin-overexpressing cells (For example, tumor vascular endothelia), an anti-angiogenic drug (DOX), a vascular disrupting agent (fosbretabulin) and a gold nanorod. This enabled both drug delivery and PTT in a murine fibrosarcoma xenograft model and the results indicated a decrease in existing vasculature and the inhibition of neovascularization within tumors (57). Furthermore, delivery of interleukin-12 in RGD-peptide AuNPs may enhance adoptive T-cell therapy and improve its therapeutic potential (58). Neuropilin (Nrp)-1 receptor-specific short peptide-coated cucurbituril AuNPs deliver and release curcumin in Nrp-1-overexpressing melanoma cells (59). AuNPs coated with an anti-HER2 monoclonal antibody (trastuzumab)-cytotoxic drug [monomethyl auristatin $\mathrm{E}$ (MMAE)] conjugate and a cell-penetrating peptide (HIV Tat) have been indicated to target HER-2-positive cells, increase cellular uptake and enhance the antimitotic potency and therapeutic index of free MMAE in vitro (60). Anti-EGFR-coated 5 -fluorouracil-AuNPs have also been revealed to target colorectal cancer cells that overexpress EGFR, increase their apoptosis rate and improve their anti-tumor effects (9). DOX and anti-PD-L1 antibody-conjugated AuNPs enable drug delivery and PTT to target and induce CT-26 cellular apoptosis and inhibit cell proliferation (9). Furthermore, the attachment of glucose to polyethylene glycol (PEG) at the C6 position followed by installation on sub-50-nm nanocarrier enhances the delivery of polo-like kinase 1 siRNA to cancer stem-like cells, which overexpress glucose transporter 1 on their surface (61).

By carrying drugs in combination with specific nucleic acid sequences, short peptides, antibodies and receptors, AuNPs can aggregate at target tissues and cells to modulate drug release rate and improve efficacy.

\section{Further innovations}

Eco-friendly AuNPs. Protection of the environment has received increasing attention in recent years, and the use of eco-friendly materials has been investigated regarding the synthesis of AuNPs. The flower bud extract of Tussilago farfara has been used as a reducing agent to synthesize AuNPs that are suitable for anticancer drug delivery (62). Photosynthesized AuNPs from Catharanthus roseus (CR) leaf extract have also been studied, as CR is a plant that is used in traditional Chinese medicine (63). Without the addition of a chemotherapeutic or siRNA therapeutic payload, these AuNPs inhibit tumor proliferation by inducing mitochondrial-mediated apoptosis via reactive oxygen species (Fig. 1) (63). In addition, leaf extracts of Ziziphus zizyphus, Coleus aromaticus, Indigofera tinctoria, Bauhinia purpurea and mulberry have been used to synthesize environmentally friendly AuNPs (64-68). 


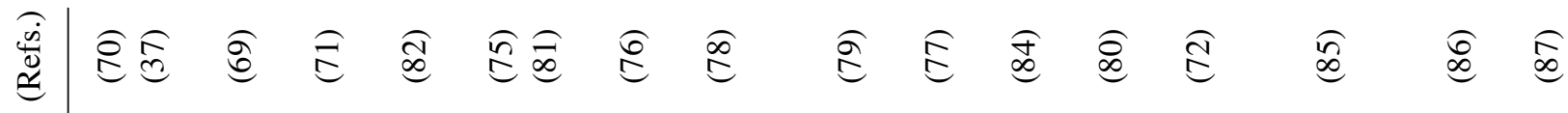

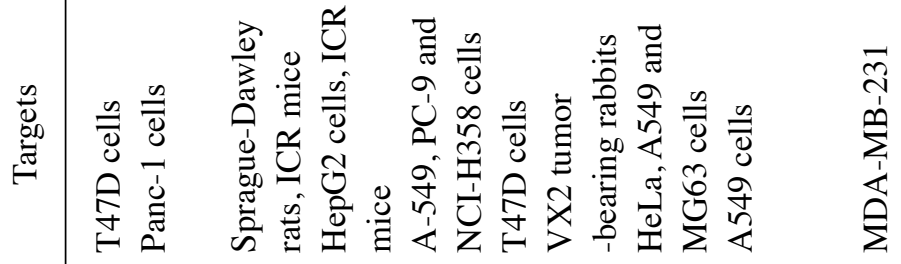

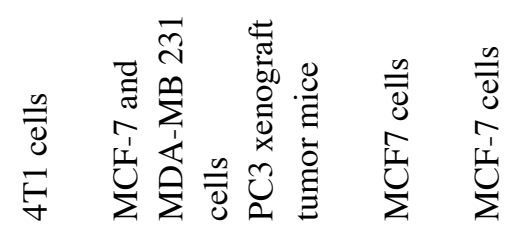

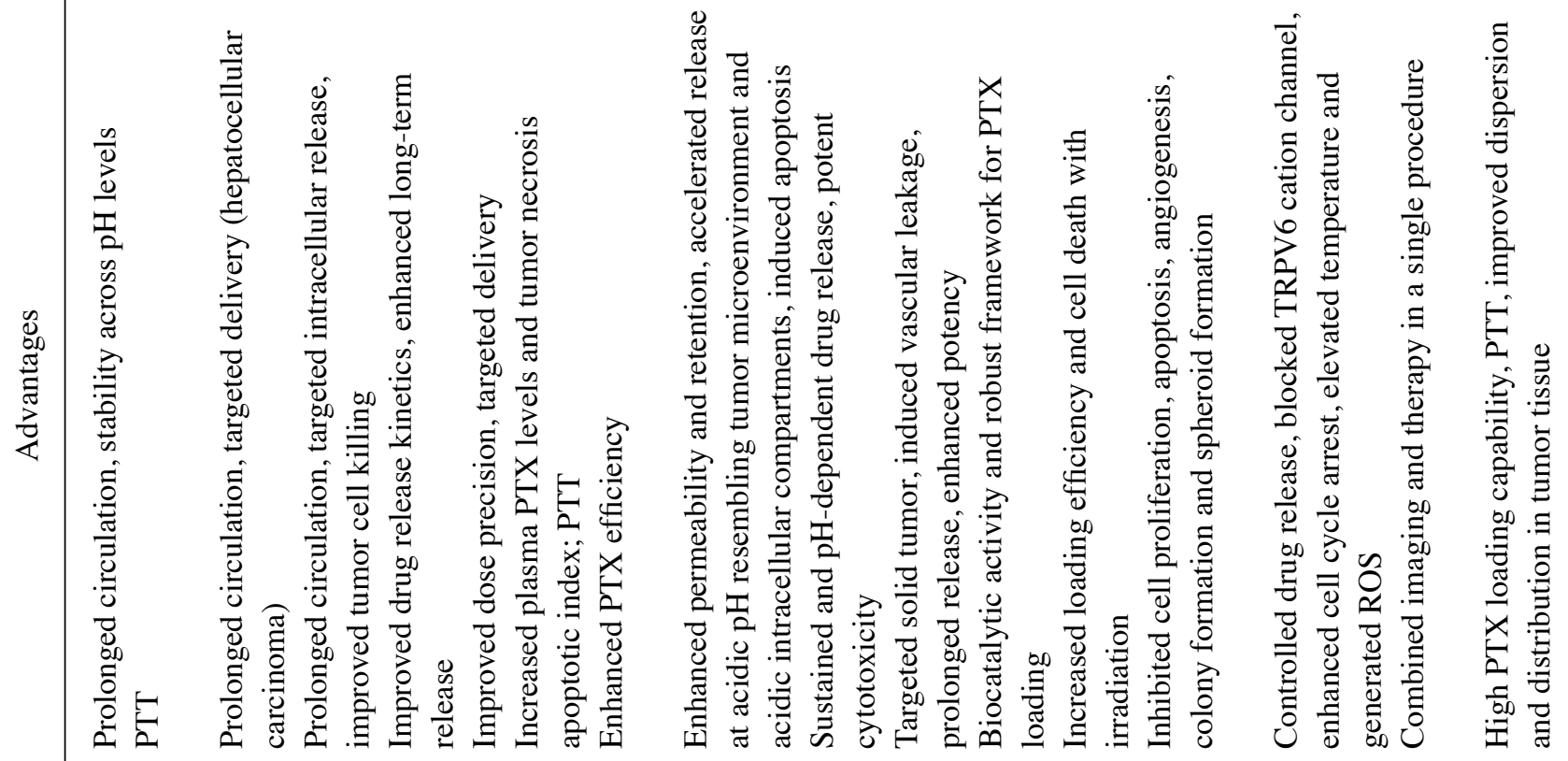

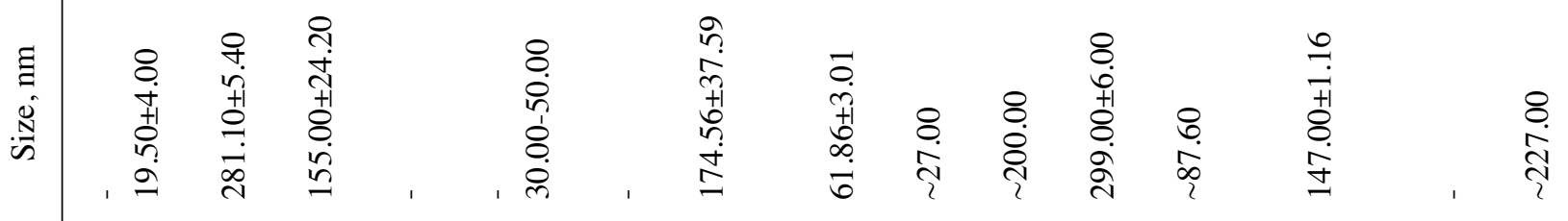

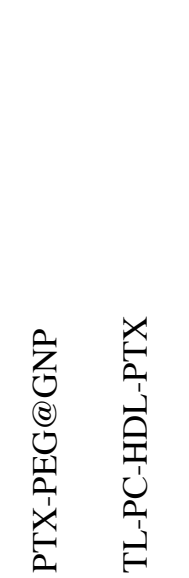

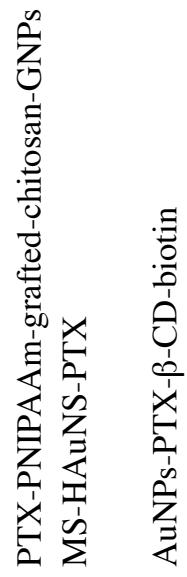

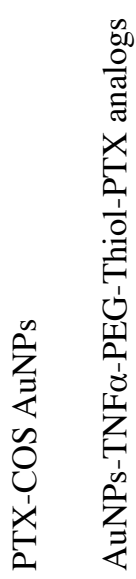

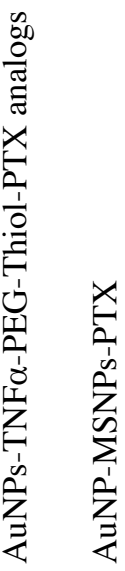

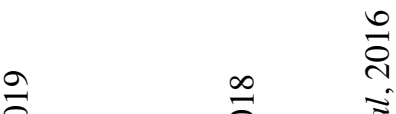

ते

$+8$

ำ

6

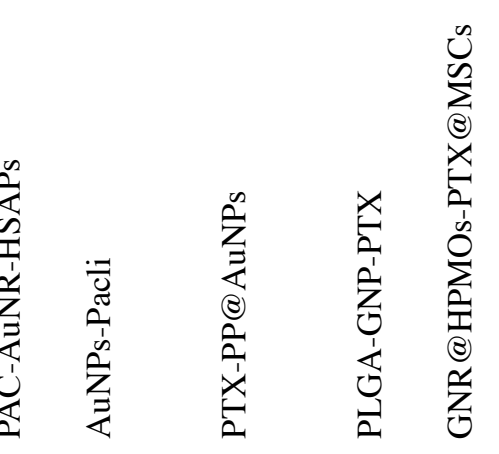

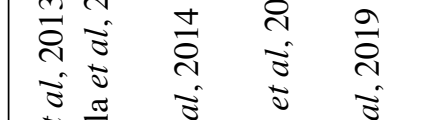


Function of paclitaxel-carrying AuNPs. As the first-line chemotherapeutic agent for lung and ovarian cancer, paclitaxel has been evaluated as a payload for multiple types of NPs, including FA/polylactic-co-glycolic acid (PLGA) NPs and hyaluronic acid-coated paclitaxel-nanostructure lipid carriers $(37,69,70)$. AuNPs are emerging as the paclitaxel carrier of choice because they are easy to synthesize across a range of sizes, absorb NIR, are highly biocompatible and are non-toxic. Table I outlines multiple paclitaxel-AuNP constructs that include nanoshells and nanorods that are added to frame materials, including PLGA, $\beta$-cyclodextrin $(\beta-C D)$ and PEG, and are modified by thiol, chitosan and FA. Multi-NPs exhibit higher paclitaxel loading capability, enhanced cytotoxicity to cancer cells, decreased toxicity to normal cells, longer circulation time and improved targeting to tumor cells (69-76). PEG and gold nanoparticles (GNP) hybrid systems may solve the solubility and stability issues of AuNPs and increase their loading capacity $(69,70)$. Conjugating AuNPs and paclitaxel via covalent bonding results in the attachment of $\sim 70$ molecules of paclitaxel per NP, facilitating enhanced antiproliferative and pro-apoptotic potency $(71,72)$. AuNP delivery of paclitaxel has been previously demonstrated to prevent P-glycoprotein-mediated multi-drug efflux in $\mathrm{H} 460$ cells that is induced by exposure to drugs without nanoparticle carriers (73). A novel drug delivery strategy has been demonstrated by the design of NPs containing perfluorohexane (PFH), gold nanorods and paclitaxel, where FA was added to target malignant cells that overexpressed FA receptors (74). A cell culture study indicated that upon laser irradiation, $\mathrm{PFH}$ is vaporized, resulting in rapid intracellular drug release and apoptosis (74). Proof-of-concept was further demonstrated in vivo (74). Poly(e-caprolactonediol)-based polyurethane/poly (N-isopropylacrylamide)-grafted chitosan core-shell nanofibers exhibit $\mathrm{pH} /$ temperature dual-responsive activity and have been indicated to be highly active against breast cancer cells (75). Hybrid AuNPs coated with PEG, biotin (a growth promoter targeting biotin receptor-overexpressing cancer cells), paclitaxel and rhodamine B-linked $\beta$-CD (to improve paclitaxel solubility) exhibit increased cellular uptake and cytotoxicity in cancer cells, without toxicity to normal cells (76). The potential of AuNPs to deliver cytokine TNF has gained increasing attention. In addition to promoting apoptosis, TNF disrupts tumor vasculature and causes vascular leakage, which increases local delivery of systemically administered chemotherapy and also sensitizes the adjacent tumor to radiation and thermal-based therapies (77). Furthermore, AuNPs carrying TNF and paclitaxel analogs are more potent than free paclitaxel in vivo (77). CYT-21625, which is a PEG-Thiol gold NP carrying paclitaxel analog 5 and TNF, is stable in plasma and enhances drug delivery by a reductive cleavage mechanism that releases native paclitaxel into the tumor microenvironment (77). In B16/F10 tumor-burdened mice, CYT-21625 improves paclitaxel delivery to tumors and increases pharmacokinetic exposure compared with free paclitaxel and paclitaxel analog 5 (77). The dose of free paclitaxel required to exert a similar anti-tumor effect was demonstrated to be 16 fold-higher than CYT-21625 (77).

Targeted pH-triggered NPs have been synthesized by combining FA and pH-sensitive poly(2-vinylpyridine)-basedpoly(ethylene oxide) with mercaptooctanoic acid (MOA) (78). 
These AuNPs are stable in the normal physiological environment, efficiently penetrate targeted tumor cells and quickly release their payload in the acidic tumor environment $(78,79)$. B33-AuMOA-FA-paclitaxel NPs exhibit enhanced permeability, retention and prolonged circulation, and efficiently target tumor cells and induce apoptosis in A549 cells (78). In addition, chitosan oligosaccharide-coated AuNPs exhibit release rates that are higher at $\mathrm{pH} 5.5(96 \%)$, and lower at $\mathrm{pH} 6.8$ and 7.4 (50-60\%). This may be associated with enhanced cytotoxicity in a more acidic microenvironment (79).

Paclitaxel-carrying AuNPs have also been indicated to amplify PTT (80). Combined with PTT, it has been indicated that microsphere-hollow Au nanosphere-paclitaxel results in enhanced tumor necrosis in a rabbit liver tumor model (81). The addition of gold nanorods to human serum albumin NPs (HSAPs) carrying paclitaxel has also been revealed to enable PTT, and NIR treatment of murine 4T1 breast cancer cells that had been treated with paclitaxel/gold rod-loaded HSAPs was observed to increase cell death from $~ 82$ to $~ 94 \%$ (80). Hybrid GNPs-Polydopamine-paclitaxel-PLGA-microspheres with NIR irradiation generate more reactive oxygen species by downregulating antioxidant enzyme expression levels, and enhancing cytotoxicity (37).

Furthermore, PTT using paclitaxel-loaded liposomes, which is modified with the addition of gold nanostars linking cyclooxygenase (COX)-2 siRNA with a targeting ligand (2-DG) and the transmembrane peptide 9-poly-D-arginine, has been identified to exhibit activity against drug-resistant cells (82). As COX-2 serves important roles in tumorigenesis, angiogenesis and the development of multi-drug resistance, its selection as a therapeutic target may yield substantial clinical benefits (82).

From 21 studies that examined paclitaxel AuNPs (Table I) (83-88), reports were identified that examined the synthesis of $\mathrm{pH}$-sensitive drug systems and conjugates of antibodies, nucleic acids and receptors to target paclitaxel delivery into specific tissues and to modulate paclitaxel release, permeability and retention.

Association between blood-brain barrier (BBB) and AuNPs. AuNPs of varying sizes may have differential uptake in different tissue types, and size may also influence BBB penetration. The BBB allows neutral, lipophilic molecules and compounds with molecular weights $<400$ Da to cross, while preventing the entry of larger toxic molecules into the brain (89). A murine study demonstrated that AuNPs with diameters of 15 and $50 \mathrm{~nm}$ were delivered to the brain and other tissues, whereas BBB penetration of 200-nm diameter AuNPs was poor (90). Moreover, AuNPs coated with exosome-derived membranes exhibit improved BBB penetration as well as improved targeting of brain neurons (91). In addition to penetrating the BBB, AuNPs may also bypass the barrier via neuronal uptake and retrograde axonal transport to the central nervous system $(92,93)$. Wheat germ agglutinin horseradish peroxidase-conjugated AuNPs deliver drugs to rat spinal cord and brainstem by bypassing the BBB via peripheral uptake and transport via the phrenic nerve (92). In a murine model of glioblastoma multiforme, polymer-coated gold-iron oxide nanoparticles carrying therapeutic miRNAs were successfully delivered to the central nervous system via intranasal administration, bypassing the BBB and augmenting the therapeutic effect of systemic temozolimide (93). In summary,
AuNPs may provide alternative drug delivery platforms by either crossing or bypassing the BBB.

\section{Limitations}

Although AuNPs offer multiple advantages as drug carriers, safety is the foremost limitation to their widespread application. The majority of reports have indicated that AuNPs are non-toxic, however, additional studies have demonstrated toxicity (94-97). Toxicity may be associated with size, shape, conjugated materials and nucleic acids, dose and biodegradability $(94,95)$. Nanostars are less toxic than nanospheres (96). In addition, surface charges and ligand types may influence toxicity. For example, cationic and polyelectrolyte-wrapped AuNPs are more toxic than electronegative and anionic 3-mercaptopropionic acid- and cationic3-mercaptopropylamine-wrapped AuNPs to Gram-negative and -positive bacteria (Shewanella oneidensis and Bacillus subtilis, respectively) (97). Based on these controversial findings, more detailed standardized criteria to evaluate AuNP toxicity are required.

\section{Conclusion}

Paclitaxel is a first-line anti-cancer drug for ovarian and breast cancer, as well as other types of solid tumor. However, poor solubility and resistance limit its widespread use. Currently, multiple types of paclitaxel NPs have been synthesized to increase efficacy, improve drug release kinetics and target specific tissues $(8,98,99)$. Among NPs of various frame materials, AuNPs are receiving increasing attention as drug delivery systems because they are not immunogenic and are generally considered to be non-toxic. Furthermore, their mass production is facilitated by their ease of synthesis and controlled sizes. Of utmost importance are the capacity of AuNPs to carry nucleic acid payloads and their unique role in PTT $(10,11)$. The current review divided AuNPs into 3 groups: Simple drug-carrying AuNPs, simple nucleic acid-carrying AuNPs and targeted AuNPs. The u use and functions of the three types were then further examined. The synthesis of paclitaxel AuNPs was subsequently discussed. Studies of paclitaxel AuNPs are increasingly focused on hybrid particles that incorporate multiple frame materials to improve solubility, prolong circulation times and enhance targeted release. Because cancer is a multigenic disease, nucleic acid-based therapy is a promising therapeutic modality that can be expedited by AuNPs $(12,45,46)$. In addition, AuNPs enable the innovative modality of synergistic chemo-PTT (44). Furthermore, because the sizes of AuNPs are easily controlled, 15 and $50 \mathrm{~nm}$ AuNPs cross the BBB and AuNPs may bypass the BBB via retrograde axonal transport, AuNPs may offer novel drug delivery platforms for the treatment of central nervous system disease $(90,92,93)$. However, the safety of AuNPs is still controversial. If accurate criteria to ensure non-toxicity can be developed, the pharmacokinetic and pharmacodynamic advances of AuNPs may enhance the therapeutic indices of paclitaxel and other cytotoxic agents, facilitate the development of gene therapy and PTT, and improve chemotherapeutic efficacy.

\section{Acknowledgements}

Not applicable. 


\section{Funding}

The present study was supported by Scientific Technology Research and Development Program of Shaanxi Province, China (grant no. 2011K12-23), and the National Science Foundation for Young Scientists of China (grant no. 81801647).

\section{Availability of data and materials}

Not applicable.

\section{Authors' contributions}

ZGW, JYD, QJJZ and SSJ designed and wrote the original manuscript. YY, LJZ, JRZ, SQZ, JJW and YZ acquired data, generated the figure and table, and reviewed and edited the manuscript. ZGW and JYD, SSJ revised the manuscript. All authors read and approved the final manuscript.

\section{Ethics approval and consent to participate}

Not applicable.

\section{Patient consent for publication}

Not applicable.

\section{Competing interests}

The authors declare that they have no competing interests.

\section{References}

1. World Health Organization (WHO): Cancer. https://www.who. int/health-topics/cancer\#tab=tab_1.

2. Ferlay J, Colombet M, Soerjomataram I, Mathers C, Parkin DM, Piñeros M, Znaor A and Bray F: Estimating the global cancer incidence and mortality in 2018: GLOBOCAN sources and methods. Int J Cancer 144: 1941-1953, 2019.

3. Fidler MM, Bray F and Soerjomataram I: The global cancer burden and human development: A review. Scand J Public Health 46: 27-36, 2018

4. Du X, Khan AR, Fu M, Ji J, Yu A and Zhai G: Current development in the formulations of non-injection administration of paclitaxel. Int J Pharm 542: 242-252, 2018.

5. Reshma PL, Unnikrishnan BS, Preethi GU, Syama HP, Archana MG, Remya K, Shiji R, Sreekutty J and Sreelekha TT: Overcoming drug-resistance in lung cancer cells by paclitaxel loaded galactoxyloglucan nanoparticles. Int J Biol Macromol 136 266-274, 2019.

6. Nakamura F, Seino M, Suzuki Y, Sakaki H, Sudo T, Ohta T, Tsutsumi S and Nagase S: Successful management of cutaneous lymphangitis carcinomatosa arising from cervical cancer with paclitaxel-cisplatin and bevacizumab combination therapy: A case report and review of the literature. J Med Case Rep 13: 328, 2019.

7. Lan YQ, Wu RP, Huang XB, Wang XL, Zhong DT, Huang CY and Song JT: Paclitaxel, oxaliplatin, 5-fluorouracil and leucovorin combination chemotherapy in patients with recurrent or metastatic gastric cancer. Tumori 104: 22-29, 2018.

8. Sofias AM, Dunne M, Storm G and Allen C: The battle of 'nano' paclitaxel. Adv Drug Deliv Rev 122: 20-30, 2017.

9. Emami F, Banstola A, Vatanara A, Lee S, Kim JO, Jeong JH and Yook S: Doxorubicin and Anti-PD-L1 antibody conjugated gold nanoparticles for colorectal cancer photochemotherapy. Mol Pharm 16: 1184-1199, 2019.

10. Abdel-Rashid RS, Omar SM, Teiama MS, Khairy A, Magdy M and Anis B: Fabrication of gold nanoparticles in absence of surfactant as in vitro carrier of plasmid DNA. Int J Nanomed 14: 8399-8408, 2019.
11. Uddin MI, Kilburn TC, Yang R, McCollum GW, Wright DW and Penn JS: Targeted imaging of VCAM-1 mRNA in a mouse model of laser-induced choroidal neovascularization using antisense hairpin-DNA-functionalized gold-nanoparticles. Mol Pharm 15: 5514-5520, 2018.

12. Chan KP, Chao SH and Kah JCY: Universal mRNA translation enhancement with gold nanoparticles conjugated to oligonucleotides with a Poly(T) sequence. ACS Appl Mater Interfaces 10: 5203-5212, 2018.

13. Cragg GM: Paclitaxel (Taxol): A success story with valuable lessons for natural product drug discovery and development. Med Res Rev 18: 315-331, 1998.

14. Khanna C, Rosenberg M and Vail DM: A review of paclitaxel and novel formulations including those suitable for use in dogs. J Vet Intern Med 29: 1006-1012, 2015.

15. Ettinger DS, Wood DE, Aggarwal C, Aisner DL, Akerley W, Bauman JR, Bharat A, Bruno DS, Chang JY, Chirieac LR, et al: NCCN guidelines insights: Non-small cell lung cancer, version 1.2020. J Natl Compr Canc Netw 17: 1464-1472, 2019.

16. Goetz MP, Gradishar WJ, Anderson BO, Abraham J, Aft R, Allison KH, Blair SL, Burstein HJ, Dang C, Elias AD, et al: NCCN guidelines insights: Breast cancer, version 3.2018. J Natl Compr Canc Netw 17: 118-126, 2019.

17. Benson AB, Venook AP, Al-Hawary MM, Cederquist L, Chen YJ, Ciombor KK, Cohen S, Cooper HS, Deming D, Engstrom PF, et al: NCCN guidelines insights: Colon cancer, version 2.2018. J Natl Compr Canc Netw 16: 359-369, 2018.

18. Ajani JA, D'Amico TA, Almhanna K, Bentrem DJ, Chao J, Das P, Denlinger CS, Fanta P, Farjah F, Fuchs CS, et al: Gastric Cancer, Version 3.2016, NCCN Clinical Practice Guidelines in Oncology. J Natl Compr Canc Netw 14: 1286-1312, 2016.

19. Zhu L and Chen L: Progress in research on paclitaxel and tumor immunotherapy. Cell Mol Biol Lett 24: 40, 2019.

20. Spitz DR, Dornfeld KJ, Krishnan K and Giusthe D (eds): Oxidative stress in cancer biology and therapy. Humana Press, 2012.

21. Hsiao JR, Leu SF and Huang BM: Apoptotic mechanism of paclitaxel-induced cell death in human head and neck tumor cell lines. J Oral Pathol Med 38: 188-197, 2009.

22. Honore S, Kamath K, Braguer D, Horwitz SB, Wilson L, Briand $\mathrm{C}$ and Jordan MA: Synergistic suppression of microtubule dynamics by discodermolide and paclitaxel in non-small cell lung carcinoma cells. Cancer Res 64: 4957-4964, 2004.

23. Abu Samaan TM, Samec M, Liskova A, Kubatka P and Büsselberg D: Paclitaxel's mechanistic and clinical effects on breast cancer. Biomolecules 9: 789, 2019.

24. Ai B, Bie Z, Zhang S and Li A: Paclitaxel targets VEGF-mediated angiogenesis in ovarian cancer treatment. Am J Cancer Res 6: 1624-1635, 2016.

25. Nakajima T, Elovaara E, Gonzalez FJ, Gelboin HV, Raunio H, Pelkonen O, Vainio H and Aoyama T: Styrene metabolism by cDNA-expressed human hepatic and pulmonary cytochromes P450. Chem Res Toxicol 7: 891-896, 1994.

26. van Eijk M, Boosman RJ, Schinkel AH, Huitema ADR and Beijnen JH: Cytochrome P450 3A4, 3A5, and 2C8 expression in breast, prostate, lung, endometrial, and ovarian tumors: Relevance for resistance to taxanes. Cancer Chemother Pharmacol 84: 487-499, 2019.

27. Noll EM, Eisen C, Stenzinger A, Espinet E, Muckenhuber A, Klein C, Vogel V, Klaus B, Nadler W, Rösli C, et al: CYP3A5 mediates basal and acquired therapy resistance in different subtypes of pancreatic ductal adenocarcinoma. Nat Med 22: 278-287, 2016

28. Němcová-Fürstová V, Kopperová D, Balušíková K, Ehrlichová M, Brynychová M, Václavíková R, Daniel P, Souček P and Kovář J: Characterization of acquired paclitaxel resistance of breast cancer cells and involvement of ABC transporters. Toxicol Appl Pharmacol 310: 215-228, 2016.

29. Njiaju UO, Gamazon ER, Gorsic LK, Delaney SM, Wheeler HE, Im HK and Dolan ME: Whole-genome studies identify solute carrier transporters in cellular susceptibility to paclitaxel. Pharmacogenet Genomics 22: 498-507, 2012.

30. Cao X, Hou J, An Q, Assaraf YG and Wang X: Towards the overcoming of anticancer drug resistance mediated by 553 mutations. Drug Resist Updat 49: 100671, 2019.

31. Xu JH, Hu SL, Shen GD and Shen G: Tumor suppressor genes and their underlying interactions in paclitaxel resistance in cancer therapy. Cancer Cell Int 16: 13, 2016.

32. Boraschi D, Italiani P, Palomba R, Decuzzi P, Duschl A, Fadeel B and Moghimi SM: Nanoparticles and innate immunity: New perspectives on host defence. Semin Immunol 34: 33-51, 2017. 
33. Primard C, Rochereau N, Luciani E, Genin C, Delair T, Paul S and Verrier B: Traffic of poly(lactic acid) nanoparticulate vaccine vehicle from intestinal mucus to sub-epithelial immune competent cells. Biomaterials 31: 6060-6068, 2010.

34. Ashour AE, Badran M, Kumar A, Hussain T, Alsarra IA and Yassin AEB: Physical PEGylation enhances the cytotoxicity of 5-fluorouracil-loaded PLGA and PCL nanoparticles. Int J Nanomedicine 14: 9259-9273, 2019.

35. Yang $\mathrm{S}$ and $\mathrm{Gao} \mathrm{H}$ : Nanoparticles for modulating tumor microenvironment to improve drug delivery and tumor therapy. Pharmacol Res 126: 97-108, 2017.

36. Wang Y, Xie Y, Li J, Peng ZH, Sheinin Y, Zhou J and Oupický D Tumor-penetrating nanoparticles for enhanced anticancer activity of combined photodynamic and hypoxia-activated therapy. ACS Nano 11: 2227-2238, 2017.

37. Banstola A, Pham TT, Jeong JH and Yook S Polydopamine-tailored paclitaxel-loaded polymeric microspheres with adhered NIR-controllable gold nanoparticles for chemo-phototherapy of pancreatic cancer. Drug Deliv 26: 629-640, 2019

38. Zhao L, Bi D, Qi X, Guo Y, Yue F, Wang X and Han M: Polydopamine-based surface modification of paclitaxel nanoparticles for osteosarcoma targeted therapy. Nanotechnology 30 255101, 2019.

39. Li W, Cao Z, Liu R, Liu L, Li H, Li X, Chen Y, Lu C and Liu Y: AuNPs as an important inorganic nanoparticle applied in drug carrier systems. Artif Cells Nanomed Biotech 47: 4222-4233, 2019

40. Das P, Fatehbasharzad P, Colombo M, Fiandra L and Prosperi D: Multifunctional magnetic gold nanomaterials for cancer. Trends Biotech 37: 995-1010, 2019.

41. Paciotti GF, Myer L, Weinreich D, Goia D, Pavel N, McLaughlin RE and Tamarkin L: Colloidal gold: A novel nanoparticle vector for tumor directed drug delivery. Drug Deliv 11: 169-183, 2004.

42. Hale SJM, Perrins RD, Garci A CE, Pace A, Peral U, Patel KR Robinson A, Williams P, Ding Y, Saito G, et al: DM1 loaded ultrasmall gold nanoparticles display significant efficacy and improved tolerability in murine models of hepatocellular carcinoma. Bioconjug Chem 30: 703-713, 2019.

43. Govindaraju S, Roshini A, Lee MH and Yun K: Kaempferol conjugated gold nanoclusters enabled efficient for anticancer therapeutics to A549 lung cancer cells. Int J Nanomedicine 14 5147-5157, 2019.

44. Zhang X, Liu Y, Luo L, Li L, Xing S, Yin T, Bian K, Zhu R and Gao D: A chemo-photothermal synergetic antitumor drug delivery system: Gold nanoshell coated wedelolactone liposome. Mater Sci Eng C Mater Biol Appl 101: 505-512, 2019.

45. Tortiglione C and de la Fuente JM: Synthesis of gold nanoparticles for gene silencing. Methods Mol Biol 1974: 203-214, 2019.

46. Wang J, Thomas M, Lin P, Cheng JX, Matei DE and Wei A siRNA delivery using dithiocarbamate-anchored oligonucleotides on gold nanorods. Bioconjug Chem 30: 443-453, 2019.

47. Ye W, Li H, Li X, Fan X, Jin Q and Ji J: mRNA guided intracellular self-assembly of DNA-gold nanoparticle conjugates as a precise trigger to up-regulate cell apoptosis and activate photothermal therapy. Bioconjug Chem 30: 1763-1772, 2019.

48. Talamantez-Lyburn S, Brown P, Hondrogiannis N, Ratliff J, Wicks SL, Nana N, Zheng Z, Rosenzweig Z, Hondrogiannis E, Devadas MS and Ehrlich ES: Gold nanoparticles loaded with cullin-5 DNA increase sensitivity to 17-AAG in cullin-5 deficient breast cancer cells. Int J of Pharm 564: 281-292, 2019.

49. Liu B, Cao W, Qiao G, Yao S, Pan S, Wang L, Yue C, Ma L, Liu Y and Cui D: Effects of gold nanoprism-assisted human PD-L1 siRNA on both gene down-regulation and photothermal therapy on lung cancer. Acta Biomater 99: 307-319, 2019.

50. Sukumar UK, Bose RJC, Malhotra M, Babikir HA, Afjei R, Robinson E, Zeng Y, Chang E, Habte F, Sinclair R, et al: Intranasal delivery of targeted polyfunctional gold-iron oxide nanoparticles loaded with therapeutic microRNAs for combined theranostic multimodality imaging and presensitization of glioblastoma to temozolomide. Biomaterials 218 $119342,2019$.

51. Zhang J, Zhao T, Han F, Hu Y and Li Y: Photothermal and gene therapy combined with immunotherapy to gastric cancer by the gold nanoshell-based system. J Nanobiotechnology 17 : $80,2019$.

52. Chen X, Han W, Zhao XAO, Tang W and Wang F: Epirubicin-loaded marine carrageenan oligosaccharide capped gold nanoparticle system for $\mathrm{pH}$-triggered anticancer drug release. Sci Rep 9: 6754, 2019.
53. Pedrosa P, Corvo ML, Margarida FS, Martins P, Carvalheiro MC, Costa PM, Martins C, Martins LMDRS, Baptista PV and Fernandes AR: Targeting cancer resistance via multifunctional gold nanoparticles. Int J Mol Sci 20: 5510, 2019.

54. Cryer AM, Chan C, Eftychidou A, Maksoudian C, Mahesh M, Tetley TD, Spivey AC and Thorley AJ: Tyrosine kinase inhibitor gold nanoconjugates for the treatment of non-small cell lung cancer. ACS App Mat Interfaces 11: 16336-16346, 2019.

55. Zhang H, Cui W, Qu X, Wu H, Qu L, Zhang X, Mäkilä E, Salonen J, Zhu Y, Yang Z, et al: Photothermal-responsive nanosized hybrid polymersome as versatile therapeutics codelivery nanovehicle for effective tumor suppression. Proc Nat Acad Sci USA 116: 7744-7749, 2019.

56. Paris JL, Villaverde G, Gómez-Graña S and Vallet-Regí M: Nanoparticles for multimodal antivascular therapeutics: Dual drug release, photothermal and photodynamic therapy. Acta Biomater 101: 459-468, 2020.

57. Gasparri AM, Sacch A, Basso V, Cortesi F, Freschi M, Rrapaj E, Bellone M, Casorati G, Dellabona P, Mondino A, et al: Boosting interleukin-12 antitumor activity and synergism with immunotherapy by targeted delivery with isoDGR-tagged nanogold. Small 15: e1903462, 2019.

58. Barman S, Das G, Gupta V, Mondal P, Jana B, Bhunia D, Khan J, Mukherjee D and Ghos S: Dual-arm nanocapsule targets neuropilin-1 receptor and microtubule: A potential nanomedicine platform. Mol Pharm 16: 2522-2531, 2019.

59. Cruz E and Kayser V: Synthesis and enhanced cellular uptake in vitro of Anti-HER2 multifunctional gold nanoparticles. Cancers (Basel) 11: 870, 2019.

60. Groysbeck N, Stoessel A, Donzeau M, da Silva EC, Lehmann M, Strub JM, Cianferani S, Dembélé K and Zuber G: Synthesis and biological evaluation of $2.4 \mathrm{~nm}$ thiolate-protected gold nanoparticles conjugated to Cetuximab for targeting glioblastoma cancer cells via the EGFR. Nanotechnology 30: 184005-184005, 2019.

61. Yi Y, Kim HJ, Zheng M, Mi P, Naito M, Kim BS, Min HS Hayash K, Perche F, Toh K, et al: Glucose-linked sub-50-nm unimer polyion complex-assembled gold nanoparticles for targeted siRNA delivery to glucose transporter 1-overexpressing breast cancer stem-like cells. J Control Release 295: 268-277, 2019.

62. Lee YJ, Song K, Cha SH, Cho S, Kim YS and Park Y: Sesquiterpenoids from Tussilago farfara flower bud extract for the eco-friendly synthesis of silver and gold nanoparticles possessing antibacterial and anticancer activities. Nanomaterials (Basel) 9: 819, 2019

63. Boomi P, Ganesan RM, Poorani G, Gurumallesh Prabu H, Ravikumar S and Jeyakanthan J: Biological synergy of greener gold nanoparticles by using Coleus aromaticus leaf extract. Mater Sci Eng C Mater Biol Appl 99: 202-210, 2019.

64. Vijayan R, Joseph S and Mathew B: Indigofera tinctoria leaf extract mediated green synthesis of silver and gold nanoparticles and assessment of their anticancer, antimicrobial, antioxidant and catalytic properties. Artif Cells Nanomed Biotechnol 46: 861-871, 2018.

65. Aljabali AAA, Akkam Y, Al Zoubi MS, Al-Batayneh KM, Al-Trad B, Abo Alrob O, Alkilany AM, Benamara M and Evans DJ: Synthesis of Gold Nanoparticles Using Leaf Extract of Ziziphus zizyphus and their Antimicrobial Activity. Nanomaterials (Basel) 8: 174, 2018.

66. Xu L, Li W, Shi Q, Li H, Yang Z, Liao D, Li L, Yang X and Zhang J: Synthesis of mulberry leaf extract mediated gold nanoparticles and their ameliorative effect on Aluminium intoxicated and diabetic retinopathy in rats during perinatal life. J Photochem Photobiol B 196: 111502, 2019.

67. Ke Y, Al Aboody MS, Alturaiki W, Alsagaby SA, Alfaiz FA, Veeraraghavan VP and Mickymaray S: Photosynthesized gold nanoparticles from Catharanthus roseus induces caspase-mediated apoptosis in cervical cancer cells (HeLa). Artif Cells Nanomed Biotechnol 47: 1938-1946, 2019.

68. Vijayan R, Joseph S and Mathew B: Anticancer, antimicrobial, antioxidant, and catalytic activities of green-synthesized silver and gold nanoparticles using Bauhinia purpurea leaf extract. Bioprocess Biosyst Eng 42: 305-319, 2019.

69. Bao QY, Zhang N, Geng DD, Xue GW, Merritt M, Zhang C and Ding Y: The enhanced longevity and liver targetability of Paclitaxel by hybrid liposomes encapsulating paclitaxel-conjugated gold nanoparticles. Int J Pharm 477: 408-415, 2014.

70. Ding Y, Zhou YY, Chen H, Geng DD, Wu DY, Hong J, Shen WB, Hang TG and Zhang C, The performance of thiol-terminated PEG-paclitaxel-conjugated gold nanoparticles. Biomaterials 34: 10217-10227, 2013 
71. Gibson JD, Khanal BP, and Zubarev ER: Paclitaxel-functionalized gold nanoparticles. J Am Chem Soc 129: 11653-11661, 2007.

72. Vemuri SK, Banala RR, Mukherjee S, Uppula P, Gpv S, A V GR and T M: Novel biosynthesized gold nanoparticles as anti-cancer agents against breast cancer: Synthesis, biological evaluation, molecular modelling studies. Mater Sci Eng C Mater Biol Appl 99: 417-429, 2019.

73. Li F, Zhou XF, Zhou HY, Jia JB, Li LW, Zhai SM and Yan B: Reducing both Pgp overexpression and drug efflux with anti-cancer gold-paclitaxel nanoconjugates. PLoS One 11: e0160042, 2016.

74. Zhong J, Yang S, Wen L and Xing D: Imaging-guided photoacoustic drug release and synergistic chemo-photoacoustic therapy with paclitaxel-containing nanoparticles. J Control Release 226: 77-87, 2016

75. Farboudi A, Nouri A, Shirinzad S, Sojoudi P, Davaran S, Akrami M and Irani M: Synthesis of magnetic gold coated poly (epsilon-caprolactonediol) based polyurethane/poly(N-isopropyl acrylamide)-grafted-chitosan core-shell nanofibers for controlled release of paclitaxel and 5-FU. Int J Biol Macromol 150 1130-1140, 2020

76. Heo DN, Yang DH, Moon HJ, Lee JB, Bae MS, Lee SC, Lee WJ, Sun IC and Kwon IK: Gold nanoparticles surface-functionalized with paclitaxel drug and biotin receptor as theranostic agents for cancer therapy. Biomaterial 33: 856-866, 2012.

77. Paciotti GF, Zhao JL, Cao SG, Brodie PG, Tamarkin L, Huhta M, Myer LD, Friedman J and Kingston DG: Synthesis and evaluation of paclitaxel-loaded gold nanoparticles for tumor-targeted drug delivery. Bioconjug Chem 27: 2646-2657, 2016.

78. Liaskoni A, Angelopoulou A, Voulgari E, Popescu MT, Tsitsilianis $\mathrm{C}$ and Avgoustakis K: Paclitaxel controlled delivery using a $\mathrm{pH}$-responsive functional-AuNP/block-copolymer vesicular nanocarrier composite system. Eur J Pharm Sci 117: 177-186, 2018

79. Manivasagan $\mathrm{P}$, Bharathiraja S, Bui NQ, Lim IG and Oh JH: Paclitaxel-loaded chitosan oligosaccharide-stabilized gold nanoparticles as novel agents for drug delivery and photoacoustic imaging of cancer cells. Int J Pharm 511: 367-379, 2016.

80. Peralta DV, Heidari Z, Dash S and Tarr MA: Hybrid paclitaxel and gold nanorod-loaded human serum albumin nanoparticles for simultaneous chemotherapeutic and photothermal therapy on 4T1 breast cancer cells. ACS Appl Mater Interfaces 7: 7101-7111, 2015.

81. Gupta S, Stafford RJ, Javadi S, Ozkan E, Ensor JE, Wright KC, Elliot AM, Jian Y, Serda RE, Dixon KA, et al: Effects of near-infrared laser irradiation of biodegradable microspheres containing hollow gold nanospheres and paclitaxel administered intraarterially in a rabbit liver tumor model. J Vasc Interv Radiol 23: 553-561, 2012.

82. Zhu HY, Han WL, Gan Y, Li QF, Li XL, Shao LL, Zhu D and Guo HW: Combined modality therapy based on hybrid gold nanostars coated with temperature sensitive liposomes to overcome paclitaxel-resistance in hepatic carcinoma. Pharmaceutics 11: 683, 2019.

83. England CG, Miller MC, Kuttan A, Trent JO and Frieboes HB: Release kinetics of paclitaxel and cisplatin from two and three layered gold nanoparticles. Eur J Pharm Biopharm 92: 120-129, 2015.

84. Pandey PC, Pandey G and Narayan RJ: Polyethyleniminemediated synthetic insertion of gold nanoparticles into mesoporous silica nanoparticles for drug loading and biocatalysis. Biointerphases 12: 011005, 2017.
85. Wang Q, Zhang X, Sun Y, Wang L, Ding L, Zhu WH, Di W and Duan YR: Gold-caged copolymer nanoparticles as multimodal synergistic photodynamic/photothermal/chemotherapy platform against lethality androgen-resistant prostate cancer. Biomaterials 212: 73-86, 2019.

86. Yahyaei B and Pourali P: One step conjugation of some chemotherapeutic drugs to the biologically produced gold nanoparticles and assessment of their anticancer effects. Sci Rep 9: 10242, 2019.

87. Wu J, Liu Y, Tang YX, Wang SJ, Wang CY, Li YJ, Su XD, Tian JH, Tian Y, Pan J, et al: Synergistic chemo-photothermal therapy of breast cancer by mesenchymal stem cell-encapsulated yolk-shell GNR@HPMO-PTX nanospheres. ACS Appl Mater Interfaces 8: 17927-17935, 2016.

88. You J, Wang Z, Du Y, Yuan H, Zhang P, Zhou J, Liu F, Li C and $\mathrm{Hu} F$ : Specific tumor delivery of paclitaxel using glycolipid-like polymer micelles containing gold nanospheres. Biomaterials 34 : 4510-4519, 2013.

89. Pardridge WM: The blood-brain barrier, bottleneck in brain drug development. NeuroRx 2: 3-14, 2005.

90. Sonavane G, Tomoda K and Makino K: Biodistribution of colloidal gold nanoparticles after intravenous administration, effect of particle size. Colloids Surf B Biointerfaces 66: 274-280, 2008.

91. Khongkow M, Yata T, Boonrungsiman S, Ruktanonchai UR, Graham D and Namdee K: Surface modification of gold nanoparticles with neuron-targeted exosome for enhanced blood-brain barrier penetration. Sci Rep 9: 8278, 2019.

92. Zhang Y, Walker JB, Minic Z, Liu F, Goshgarian H and Mao G: Transporter protein and drug-conjugated gold nanoparticles capable of bypassing the blood-brain barrier. Sci Rep 6: 25794, 2016.

93. Singh N, Nayak J, Sahoo SK and Kumar R: Glutathione conjugated superparamagnetic $\mathrm{Fe}_{3} \mathrm{O}_{4}$-Au core shell nanoparticles for $\mathrm{pH}$ controlled release of DOX. Mater Sci Eng C Mater Biol App 100: 453-465, 2019.

94. Gao W, Xu K, Ji L and Tang B: Effect of gold nanoparticles on glutathione depletion-induced hydrogen peroxide generation and apoptosis in HL7702 cells. Toxicol Lett 205: 86-95, 2011.

95. Coradeghini R, Gioria S, García CP, Nativo P, Franchini F, Gilliland D, Ponti J and Rossi F: Size-dependent toxicity and cell interaction mechanisms of gold nanoparticles on mouse fibroblasts. Toxicol Lett 217: 205-216, 2013.

96. Favi PM, Gao M, Johana Sepúlveda Arango L, Ospina SP, Morales M, Pavon JJ and Webster TJ: Shape and surface effects on the cytotoxicity of nanoparticles: Gold nanospheres versus gold nanostars. J Biomed Mater Res A 103: 3449-3462, 2015.

97. Feng ZV, Gunsolus IL, Qiu TA, Hurley KR, Nyberg LH, Frew H, Johnson KP, Vartanian AM, Jacob LM, Lohse SE, et al: Impacts of gold nanoparticle charge and ligand type on surface binding and toxicity to Gram-negative and Gram-positive bacteria. Chem Sci 6: 5186-5196, 2015.

98. Gorain B, Choudhury H, Pandey M and Kesharwani P: Paclitaxel loaded vitamin E-TPGS nanoparticles for cancer therapy. Mater Sci Eng C Mater Biol Appl 91: 868-880, 2018.

99. Kundranda MN and Niu J: Albumin-bound paclitaxel in solid tumors: Clinical development and future directions. Drug Des Devel Ther 9: 3767-3777, 2015.

(i) (5) This work is licensed under a Creative Commons Attribution-NonCommercial-NoDerivatives 4.0 International (CC BY-NC-ND 4.0) License. 